\section{PWE-007 UPTAKE OF THE BOWEL CANCER SCREENING PROGRAMME IN THE EASTERN REGION OF ENGLAND: TRENDS BY AGE AND GENDER}

doi:10.1136/gutjnl-2013-304907.296

\begin{abstract}
1."A Field, 'M Vogler, 'R F Logan. 'Eastern Bowel Cancer Screening Hub, Nottingham
\end{abstract} University Hospitals Trust, Nottingham, UK

Introduction Bowel cancer is more common in men than women with age-adjusted incidence in the UK being over $50 \% \%$ higher in men and mortality $63 \%$ higher. However uptake of the Bowel Cancer Screening Programme in England (BCSP) has been generally about $5 \%$ lower in men ${ }^{1}$. We have examined whether with increasing familiarity with the BCSP there have been changes in uptake of screening invitations over the past $4-5$ years.

Methods Analysis of uptake of screening invitations by sex and 2 year age bands of people invited for screening by the Eastern Hub which covers the East of England and East Midlands (total population 10.6 million) between 1 Jan 2008 and 30 June 2012 by 6 month periods.

Results Over the 4.5 years $>2.8$ million invitations were sent by the Eastern Hub. For those aged 60-61 years uptake by men was 9-10\% lower than that by women (uptake $60 \%$ ) with no evidence of any change over the 4 years. In women uptake remained over $60 \%$ for the 62-63, 64-65 and 66-67 age bands with a small increase over time reaching $65 \%$ in 2012 . In men uptake showed a steady increase with age such that by age 68-69 uptake was only $2-3 \%$ lower than in women. For those aged 70-71 uptake in men and women was generally similar and for those aged 72-73 and those aged 74 uptake was around $2 \%$ higher in men than women. This was partly accounted for by a decline in uptake in women to below $60 \%$. Analysis of time trends in those over 70 was unreliable because of only partial roll-out ('age extension') of the BCSP to this group in the Eastern region.

Conclusion While the small decline in uptake in women $>70$ years is a concern the increasing uptake in men with age is encouraging and suggests that the introduction of simpler screening approaches using faecal immunochemical tests will see substantial increses in uptake of the $\mathrm{BCSP}^{2}$

Disclosure of Interest None Declared.

\section{REFERENCES}

1. Logan RF, Patnick J, Nickerson C, et al. Outcomes of the Bowel Cancer Screening Programme in England after the first 1 million tests. Gut 2012, vol 61, pp1439-46.

2. van Rossum LG, van Rijn AF, Laheij RJ et al. Random comparison of guaiac and immunochemical faecal occult blood tests for colorectal cancer in a screening population Gastroenterology 2008 vol 135 pp82-90

\section{PWE-008 PLACIDE: PROBIOTICS IN THE PREVENTION OF ANTIBIOTIC ASSOCIATED DIARRHOEA (AAD) AND CLOSTRIDIUM DIFFICILE ASSOCIATED DIARRHOEA (CDD) IN ELDERLY PATIENTS ADMITTED TO HOSPITAL - RESULTS OF A LARGE MULTI-CENTRE RCT IN THE UK}

doi:10.1136/gutjnl-2013-304907.297

${ }^{1} \mathrm{~S}$ Allen, ${ }^{2 \mathrm{~K}}$ Wareham, ${ }^{3} \mathrm{D}$ Wang, ${ }^{4} \mathrm{C}$ Bradley, ${ }^{5} \mathrm{~B}$ Sewell, ${ }^{1} \mathrm{H}$ Hutchings, ${ }^{6} \mathrm{~W}$ Harris, ${ }^{7 *} \mathrm{~A}$ Dhar, ${ }^{8} \mathrm{H}$ Brown, ${ }^{8} \mathrm{~A}$ Foden, ${ }^{9} \mathrm{M}$ Gravenor, ${ }^{10} \mathrm{~S}$ Plummer, ${ }^{9} \mathrm{D}$ Mack, ${ }^{5} \mathrm{C}$ J Phillips. ${ }^{1}$ Paediatrics, College of Medicine; ${ }^{2}$ Clinical Research Unit, Morriston Hospital, Swansea University, Swansea; ' London School of Hygiene and Tropical Medicine, London; ${ }^{4}$ Pharmacy, County Durham \& Darlington NHS Foundation Trust, Darlington; ${ }^{5}$ Swansea Centre for Health Economics, Swansea University, Swansea; ${ }^{6}$ Abertawe Bro Morgannwg University Hospital Board, Port Talbot; ${ }^{7}$ Gastroenterology; ${ }^{8}$ Medicine \& Elderly Care, County Durham \& Darlington NHS Foundation Trust, Darlington; ${ }^{9}$ College of Medicine, Swansea University, Swansea; ${ }^{10}$ Obsidian Research Limited, Port Talbot, UK

Introduction Antibiotic associated diarrhoea (AAD) and Clostridium difficile associated diarrhoea (CDD) occur in 5-39\% of pts exposed to antibiotics. Despite previous papers proposing a beneficial role of probiotics, there has been no large randomised controlled trial evaluating their preventative effect.

Methods This large RCT funded by the HTA was aimed at finding the efficacy and cost-effectiveness of a high dose, multi-strain probiotic for prevention of $\mathrm{AAD}$ and $\mathrm{CDD}$ in older people admitted to hospital. Pts aged $>65 \mathrm{yrs}$, exposed to one or more oral/parenteral antibiotics without pre-existing diarrhoea, recent CDD or risk of probiotic adverse effects were eligible. Of 17,420 patients screened $2,981(17.1 \%)$ were recruited and allocated sequentially by a computer-generated random allocation sequence stratified by centre. $1,493(50.1 \%)$ were allocated to probiotic and 1,488(49.9\%)to placebo arm. Two strains of lactobacilli and two strains of bifidobacteria with a total of $6 \times 10^{10}$ organisms/day were taken as a daily single capsule for 21 days. The placebo was inert maltodextrin powder. Occurrence of $\mathrm{AAD}$ or $\mathrm{CDD}$ within 12 weeks of recruitment was assessed by research nurses, blinded to arm allocation.

Results ITT analysis included 2,941(98.7\%)participants. Potential risk factors for antibiotic-associated diarrhoea at baseline were similar in both arms. The frequency of $\mathrm{AAD}$ was similar in the probiotic (159/1470, 10.8\%)and placebo arms (153/1471, 10.4\%), RR: 1.04; 95\%CI0.84-1.28; $\mathrm{P}=0.72$. CDD was an uncommon cause of $\mathrm{AAD}$ and occurred in $12 / 1470(0.8 \%)$ participants in the probiotic and $17 / 1471(1.2 \%)$ in the placebo arm (RR 0.71; 95\%CI0.34-1.47; $\mathrm{P}=0.35)$.Adverse events and other outcomes were similar in both arms. Total health care costs per patient did not differ significantly between probiotics (£8020.11; 95\%CI£7622.31-£8417.90) and placebo (£8011.37; 95\%CI£7600.53-£8422.22). The incremental costeffectiveness ratio of $£ 45,636 /$ OALY was robust to changes in key parameters

Conclusion This study found no evidence that probiotic administration was effective in the elderly in preventing $\mathrm{AAD}$, although there was a trend towards reduced CDD in the probiotic arm.

Disclosure of Interest S. Allen: None Declared, K. Wareham None Declared, D. Wang: None Declared, C. Bradley: None Declared, B. Sewell: None Declared, H. Hutchings: None Declared, W. Harris: None Declared, A. Dhar Grant/Research Support from: NIHR Grant Holder, Speaker bureau with: Shire Pharmaceuticals, Warner Chilcott UK, H. Brown: None Declared, A. Foden: None Declared M. Gravenor: None Declared, S. Plummer Employee of: Research Director of Obsidian Research Limited and Director of Cultech Limited, D. Mack: None Declared, C. Phillips: None Declared

\section{PWE-009 CAECAL PH MEASUREMENT IS AN OBJECTIVE BIOMARKER OF EXCESSIVE FERMENTATION IN PATIENTS WITH BLOATING AND DISTENSION}

doi:10.1136/gutjnl-2013-304907.298

1, ${ }^{*} \mathrm{~A}$ Hobson, ${ }^{2} \mathrm{~S}$ Mohammed, ${ }^{3} \mathrm{G}$ Dukes, ${ }^{2} \mathrm{M}$ Scott. ${ }^{1} \mathrm{NDC}$, Princess Grace Hospital; ${ }^{2} \mathrm{Gl}$ Physiology, OMUL, London, UK; ${ }^{3}$ GlaxoSmithKline RGD Ltd, North Carolina, United States

Introduction Fermentation of undigested carbohydrates by the colonic microbiota is an important part of maintaining a healthy colonic environment. However, excessive fermentation is thought to play a role in exacerbating symptoms of bloating, distension, pain and alternating bowel habit. By-products of the fermentation process include short chain fatty acids (SCFA's) which reduce caecal $\mathrm{pH}$. Measurement of caecal $\mathrm{pH}$ therefore provides an opportunity to objectively quantify aspects of fermentation but has been technically challenging to achieve. In this retrospective study, we compared intestinal $\mathrm{pH}$ and pressure profiles using an ingestible wireless motility capsule (WMC) (SmartPill, Buffalo, US) in healthy controls and patients with symptoms of bloating, distension, pain and alternating bowel habit.

Methods Motility and $\mathrm{pH}$ data were reviewed from 16 healthy controls (Cx) and 16 age and sex matched patients (Px) that had undergone the WMC study using a standardised protocol. Motility 